\title{
DETERMINAÇÃO DE TEORES DE ISOFLAVONAS COMERCIALIZADAS NA FORMA DE EXTRATO SECO E MANIPULADAS POR FARMÁCIAS
}

\section{DETERMINATION OF LEVELS OF ISOFLAVONES MARKETED AS DRY EXTRACT IS MANIPULATED BY PHARMACIES}

\author{
Daniel Mantovani ${ }^{1}$; Marcos Lúcio Corazza ${ }^{2}$, Lúcio Cardozo Filho ${ }^{3}$; Nilson Marcos Tazinafo ${ }^{4}$; Marcelo \\ Ribani $^{5}$; Sílvio Cláudio da Costa $^{6}$ \\ 1;2 Universidade Federal do Paraná - Curitiba - PR. E-mail: danieluns@yahoo.com.br \\ ${ }^{3}$ Universidade Estadual de Maringá - UEM - Maringá - PR \\ ${ }^{4}$ Instituto de Tecnologia do Paraná - TECPAR - Maringá - PR \\ ${ }^{5}$ Instituto de Tecnologia do Paraná - TECPAR - Curitiba - PR \\ ${ }^{6}$ Universidade Estadual de Maringá - UEM - Maringá - PR
}

\begin{abstract}
Resumo
O consumo de produtos naturais, fitoterápicos e suplementos, contendo isoflavonas, aumentaram na última década, especialmente pelas descobertas dos efeitos benéficos causados por estes compostos associados com o alivio de sintomas, principalmente aos causados pela menopausa em mulheres que apresentam a perda hormonal. Como não há trabalhos relatando quantidades mínimas de isoflavonas nas formas glicosídicas (daidzina, glicitina e genistina) $e$ agliconas (daidzeína, gliciteína e genisteína), contidas nos extratos secos e cápsulas manipuladas por farmácias denominadas de manipulação, ou até mesmo metodologia oficial para o controle de qualidade. O presente estudo objetivou, desenvolver um método quantitativo de análise de isoflavonas em extrato seco de soja, utilizados como matérias primas, para obtenção de cápsulas fitoterápicas, comercializadas livremente sem qualquer fiscalização do comércio brasileiro. A análise quantitativa de isoflavonas em extrato seco de soja e manipulados foi realizada utilizando a cromatografia líquida de alta eficiência (CLAE) revelando grandes variações nos teores de isoflavonas, dependendo do fornecedor e manipulador. As variações ocorreram para os lotes padronizados a $40 \%$ de isoflavonas em $100 \mathrm{~g}$ de extrato e para as formas manipuladas, que são comercializadas com rótulos de 20 mg de isoflavonas. As maiores distorções nos valores foram encontrados para os compostos daidzeína 60,5\% e genisteína 451\%. Portanto, é necessário a adoção de métodos de padronização e caracterização das matérias- primas, bem como certificação das empresas comercializadoras, uma vez que esses fitoterápicos são, em muitas vezes, indicado seu consumo por profissionais da área médica.
\end{abstract}

Palavras-chave: Isoflavonas; Extrato Seco; Manipulação.

\section{Introdução}

A soja representa uma das principais cadeias produtivas do Brasil, especialmente pela grande diversidade genética, morfológica e composição nutricional além de conter em sua composição, antioxidantes e isoflavonas (MANTOVANI et al., 2011; CÉZAR et al., 2006). As 
isoflavonas fazem parte de uma ampla variedade de compostos fenólicos pertencente ao grupo dos flavonóides de baixa massa molar, denominados metabólitos secundários, as quais são produzidas pelas leguminosas com teores que variam de 0,1 a 0,5 mg/g (COWARD et al., 1993; GENOVESE; LAJOLO, 2001).

Compostos como os flavonóides integrantes das isoflavonas, alcalóides, ácidos graxos entre outros, são considerados medicamentos fitoterápicos segundo a RDC n ${ }^{\circ} 48$ de 16 de março de 2004, por apresentaram princípio ativo, devendo ser registrados conforme as normas da Agência Nacional de Vigilância Sanitária (ANVISA, 2004). Desta forma, as isoflavonas comercializadas na forma de extratos secos de soja são padronizadas com teores de $40 \%$ de isoflavonas totais, fazem parte da formulação de medicamentos fitoterápicos comercializados em todo o Brasil (CÉSAR et al., 2007). A grande demanda por esse fitoterápico veio através de estudos clínicos que enfatizaram as isoflavonas agliconas por apresentarem atividade anti-estrogênica e ainda várias propriedades biológicas benéficas à saúde humana, dentre as quais podemos destacar: atuação como antioxidante, anti-inflamatória e antimicrobiana (SETCHELL, 1998). Essas propriedades biológicas somente são ativas caso ocorra a presença das isoflavonas agliconas como a (daidzeína, gliciteína e genisteína) (MANTOVANI et al., 2009).

Porém, a grande variação dos teores de isoflavonas agliconas está intimamente relacionada aos fatores: cultivar utilizado, local de plantio, clima e tipo de solo, concluíndo que os teores de isoflavonas presentes no grão de soja sofrem influência direta destes fatores (HOECK et al. 2000).

O comércio das fontes de isoflavonas provindas especialmente do gérmen de soja e extrato de soja concentrado, são padronizados com 40\% (TURNER et al., 2007). Estudos relataram o hipocótilo do grão de soja, maiores teores de daidzina e gliciteína, enquanto que no cotilédone há teores de genistina 20 vezes maiores (ELDRIDGE e KWOLEK, 1983).

Desta forma, a Cromatografia Líquida de Alta Eficiência (CLAE) tem sido reconhecida como o método mais eficiente para determinação dos compostos de isoflavonas (SETCHELL et al., 2001). Sendo assim, o presente estudo objetivou desenvolver um método quantitativo de análise de isoflavonas em extrato seco de soja, utilizadas como matérias primas para obtenção de cápsulas fitoterápicas comercializadas livremente sem qualquer fiscalização do comércio brasileiro.

\section{Material e Métodos}

\section{Material}

Para este estudo voltado a quantificação foram utilizados os padrões: daidzina $(92,0 \%)$, glicitina $(85,3 \%)$, genistina $(85,0 \%)$, daidzeína $(99,9 \%)$, gliciteína $(99,9 \%)$ e genisteína $(97,3 \%)$; foram certificados e fornecidos pela Chromadex. 
O Reagente Metanol adquerido pela J.T. Baker, usado para dissolução da amostra e dos padrões, foi grau HPLC com 100\% de pureza e o reagente Acetonitrila fornecido pela J.T. Baker, usado para a composição do gradiente da fase móvel, foi de grau HPLC com 99,97\% de pureza. O Ácido Acético P.A., fornecido pela Merck, com $100 \%$ de pureza. Os filtros descartáveis utilizados para filtração das soluções foram fornecidos pela Millipore com 0,45 $\mu \mathrm{m}$, do tipo Millex $\mathrm{HV}$ Durapore PVDF, de 25 mm de diâmetro com água ultrapura grau 1 (ASTM D1193-91).

\section{Equipamentos}

O sistema analítico utilizado no desenvolvimento da separação das isoflavonas, foi um cromatógrafo Merck Hitachi LaChrom, composto de uma bomba quaternária L-7100, um injetor automático L-7250, um detector UV-Vis com varredura espectral L-7455, um módulo de aquecimento de colunas L-7300 e um Software Merck HSM, versão 4.1. Com utilização de uma coluna cromatográfica marca Merck, tipo LiChrospher, com dimensões de $250 \mathrm{~mm}$ x 4,6 mm d.i., RP-18; e partículas esféricas de $5 \mu \mathrm{m}$.

A balança analítica utilizada para medição de massa de padrões e extratos secos foi da marca Sartorius, modelo CP-225. Os balões volumétricos utilizados foram de 100 e $50 \mathrm{~mL}$, âmbar e pipetas volumétricas de 25; $10 ; 5$ e $2 \mathrm{~mL}$.

\section{Preparo de amostras de Extratos Secos}

Padronizou-se a pesagem com $100 \mathrm{mg}$ de extratos secos (ES), em seguida transferiu-se para um balão volumétrico de $100 \mathrm{~mL}$ e dissolvidos em metanol grau HPLC. Uma alíquota de aproximadamente $10 \mathrm{~mL}$ foi filtrada em filtro tipo Millex de $0,45 \mu \mathrm{m}$ e aproximadamente $1 \mathrm{~mL}$ foi recolhido e transferido para um vial.

\section{Preparo de amostras manipuladas}

Padronizou-se a pesagem com $15 \mathrm{mg}$ de amostra manipulada, em seguida transferiu-se para um balão volumétrico de $10 \mathrm{~mL}$ e dissolvidos em metanol grau HPLC. Uma alíquota de aproximadamente $5 \mathrm{~mL}$ foi filtrada em filtro tipo Millex de $0,45 \mu \mathrm{m}$ e aproximadamente $1 \mathrm{~mL}$ foi recolhido e transferido para um vial. 
Preparo das soluções padrões A1; A2; A3; A4 e A5

Preparou-se a solução A1 em um balão volumétrico de $100 \mathrm{~mL}$, pesando-se aproximadamente as quantidades de isoflavonas padrões dispostas na Tabela 1 e dissolvendo-se em metanol grau HPLC.

Tabela 1 - Padrões de isoflavonas em miligramas (mg) utilizadas no preparo da solução A1

\begin{tabular}{cc}
\hline Isoflavonas & Massa em mg de isoflavonas \\
\hline Daidzina & 20 \\
Glicitina & 10 \\
Genistina & 5 \\
Daidzeína & 20 \\
Gliciteína & 2 \\
Genisteína & 20 \\
\hline
\end{tabular}

As soluções padrão A2; A3; A4 e A5 foram preparadas em quatro balões volumétricos distintos, de $50 \mathrm{~mL}$ cada, pipetando-se respectivamente: 25; 10; 5 e 2 mililitros da solução A1 e os volumes de cada balão foram completados com metanol grau HPLC. Em seguida uma alíquota de aproximadamente $10 \mathrm{~mL}$ de cada uma das soluções padrão: A1; A2; A3; A4 e A5; respectivamente, filtradas separadamente, em filtro tipo Millex de $0,45 \mu \mathrm{m}$ distintos e aproximadamente $1 \mathrm{~mL}$ de cada filtrado foi recolhido separadamente em cada vial identificado. As concentrações destas soluções estão dispostas na Tabela 2.

Tabela 2 - Concentração em $\mu \mathrm{g} / \mathrm{mL}^{-1}$ dos padrões de isoflavonas com seus respectivos valores

\begin{tabular}{cccccc}
\hline Padrões & A1 & A2 & A3 & A4 & A5 \\
\hline Daidzina & 200 & 100 & 40 & 20 & 8 \\
Glicitina & 100 & 50 & 20 & 10 & 4 \\
Genistina & 50 & 25 & 10 & 5 & 2 \\
Daidzeína & 200 & 100 & 4 & 20 & 8 \\
Gliciteína & 20 & 100 & 40 & 20 & 0,8 \\
Genisteína & 200 & & & 2 & 8 \\
\hline
\end{tabular}

A1; A2; A3; A4 e A5 - são as soluções padrões de isoflavonas.

\section{Condições cromatográficas}

As injeções realizadas foram estabelecidas com $10 \mu \mathrm{L}$, realizada em condições cromatográficas numa vazão de $1,0 \mathrm{~mL} \mathrm{~min}^{-1}$ para separação das isoflavonas e otimizadas usando a fase móvel: água $+0,1 \%$ ácido acético $\mathrm{pH} 3,5(\mathrm{~S} 1)$ e a fase móvel: acetonitrila $+0,1 \%$ ácido acético pH 3,5 (S2). Um programa de gradiente foi utilizado conforme condições apresentadas na Tabela 3. 
Tabela 3 - Porcentagem (\%) das Soluções S1 e S2 respectivos tempos para obtenção do gradiente da Fase Móvel

\begin{tabular}{cll}
\hline Tempo (min) & S1 & S2 \\
\hline 0 & 86 & 14 \\
8 & 86 & 14 \\
25 & 50 & 50 \\
30 & 50 & 50 \\
33 & 86 & 14 \\
35 & 86 & 14 \\
\hline
\end{tabular}

S1- Solução de Água Ultrapura + 0,1\% de ácido acético e S2 - Solução de Acetonitrila + 0,1\% de ácido acético.

Desta forma, manteve-se a coluna cromatográfica em temperatura de $35^{\circ} \mathrm{C}$, e índice de decteção UV-vis com varredura espectral de 200 a $400 \mathrm{~nm}$, monitorado em $254 \mathrm{~nm}$, os espectros respectivamente. Três amostras de extrato seco de soja de diferentes fornecedores: 1, 2 e 3 foram preparadas em duplicata e três cromatogramas de cada amostra foram obtidos por Cromatografia Líquida de Alta Eficiência (CLAE).

\section{Resultados e Discussão}

Os valores médios obtidos em relação aos teores de isoflavonas provindas dos Fornecedores: 1, 2 e 3 continham 41,7\%, 42,0\% e 41,5\%, respectivamente. Esses valores demonstraram teores de isoflavonas totais acima dos $40 \%$ de concentração, valores acima do descriminado pelo fabricante.

Desta forma, os resultados obtidos para os respectivos extratos do Fornecedor 1 continha em sua composição 26,2\% de genisteína, com menor quantidade de gliciteína 0,22\%. E ao analisar a quantidade total dos compostos agliconas (daidzeína, gliciteína e genisteína) chegou-se a um valor de $34,0 \%$ que corresponde a um valor de $81 \%$ de todas as isoflavonas contidas no conteúdo total, presente no produto do fornecedor 1 . No entanto, ao analisar os teores de isoflavonas glicosídicas (daidzina, glicitina e genistina) somaram $7,7 \%$ do total do extrato seco.

A Figura 1; (A) mostra o perfil cromatográfico obtido para as isoflavonas contidas nas diferentes concentrações, relacionadas com a amostra de extrato seco do Fornecedor 1.

Figura 1 - Perfil cromatográfico de isoflavonas contidas em extrato seco de soja fornecedor: (A) 1-Daidzina; 2-

Glicitina; 3-Genistina; 4-Daidzeína; 5-Gliciteína e 6-Genisteína; US-Unidade de Absorbância

$\mathbf{A}$

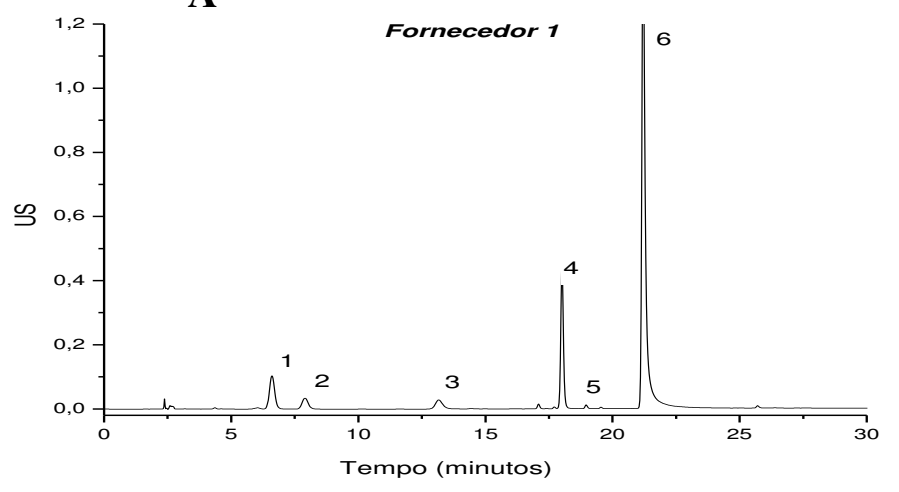


Ao estudar o Fornecedor 2 a gliciteína continuou com baixos valores $0,12 \%$ em relação ao composto glicosídico, a daidzina se destacou com 18,6\%. Porém, foi observado a inversão de valores dos compostos agliconas em relação aos glicosídicos, somando 12,9\% e 29,1\% do total de $42,0 \%$ respectivamente correspondendo a quase $70 \%$ do total de isoflavonas contidas neste lote.

No entanto, os valores obtidos do Fornecedor 3, o composto gliciteína manteve baixos valores com $0,19 \%$ ) e maior quantidade, para o composto genisteína com $21,1 \%$, com valores referentes às agliconas de $32,5 \%$ de um total de isoflavonas igual a 41,6\%, que corresponde a $78 \%$ do total das isoflavonas. Os valores referentes às isoflavonas e seus respectivos Fornecedores estão disponíveis na Tabela 4.

Tabela 4 - Média em gramas de isoflavonas por 100 gramas de extrato seco (\%) e seus respectivos desvios padrões, referentes aos três fornecedores: $1 ; 2$ e 3

\begin{tabular}{cccc}
\hline Isoflavonas & Fornecedor 1 & Fornecedor 2 & Fornecedor 3 \\
\hline Daidzina & $4,7 \pm 0,0$ & $18,6 \pm 0,2^{* *}$ & $5,6 \pm 0,1$ \\
Glicitina & $1,7 \pm 0,0$ & $7,6 \pm 0,1$ & $2,0 \pm 0,0$ \\
Genistina & $1,2 \pm 0,0$ & $2,8 \pm 0,0$ & $1,3 \pm 0,0$ \\
Daidzeína & $7,6 \pm 0,0$ & $6,9 \pm 0,0$ & $11,1 \pm 0,3$ \\
Gliciteína & $0,2 \pm 0,0^{*}$ & $0,1 \pm 0,0^{*}$ & $0,1 \pm 0,0^{*}$ \\
Genisteína & $26,2 \pm 0,0^{* *}$ & $5,8 \pm 0,0$ & $21,1 \pm 1,4^{* *}$ \\
\hline Total & $41,7 \pm 0,03$ & $42,00 \pm 0,59$ & $41,5 \pm 2,05$ \\
\hline
\end{tabular}

(*) Valores mínimos para cada fornecedor (**) Valores máximos para cada fornecedor.

As variações dos teores de isoflavonas ocorrem pela diferença de cultivares e suas diferentes condições climáticas sofridas durante o ciclo produtivo, com posterior extração da massa e padronização do teor desejado de isoflavonas (LEE, et al., 2003).

No entanto, ao estudar os compostos fitoterápicos o menor teor de isoflavonas totais foi observada para a amostra E, com valores próximos de $12 \%$ do conteúdo total, com uma grande disparidade quando comparada a amostra $\mathrm{C}$ e demais compostos analisados. Desta forma, nenhuma das amostras analisadas apresentaram teores de isoflavonas agliconas conforme indicado no rótulo ou seja, 20mg. Ao analisar quinze suplementes a base de soja, vendidos na Finlândia, o estudo demonstrou que, das quinze amostras analisadas dez estavam com valores de isoflavonas agliconas abaixo do descrito no rótulo, com teores de isoflavonas agliconas entre 23 a $69 \%$ (SONG et al., 1999).

Um estudo dirigido por Nurmi et al (2002) voltado para quantificação de suplementos à base de soja comercializados na Finlândia, foram coletadas 11 amostras das quais apenas uma estava de acordo com o rótulo. No entanto, para as demais análises realizadas a variação dos teores de isoflavonas foram entre 23 a $69 \%$ a menos do que o especificado no rótulo. 
Já para as análises de isoflavonas quantificadas em amostras manipuladas por farmácias de manipulação conforme Tabela 5, pode-se observar a maior concentração do composto daidzeína, com valores médios de até $\left.66,8 \pm 0,0 \mu \mathrm{g} \mathrm{g}^{-1}\right)$. Por outro lado, genisteína foi a isoflavona encontrada com a menor concentração, não sendo detectada nas amostras D e E.

Tabela 5 - Valores de isoflavonas para amostras obtidas de seis diferentes farmácias

\begin{tabular}{cccccc}
\hline Farmácia & Daidzina & Genistina & Daidzeína & Genisteína & Isoflavonas nível $\left(\mathbf{1 0 0} \boldsymbol{\mu g} \mathbf{g}^{-\mathbf{1}}\right)$ \\
\hline A & $8,5 \pm 0,1 \mathrm{c}$ & $7,1 \pm 0,1 \mathrm{a}$ & $3,4 \pm 0,1 \mathrm{f}$ & $16,2 \pm 0,5 \mathrm{~b}$ & $35,2 \pm 0,2 \mathrm{~d}$ \\
B & $7,0 \pm 0,5 \mathrm{c}$ & $5,2 \pm 0,1 \mathrm{c}$ & $28,3 \pm 0,3 \mathrm{~d}$ & $18,0 \pm 0,1 \mathrm{a}$ & $58,5 \pm 0,1 \mathrm{c}$ \\
C & $18,2 \pm 0,5 \mathrm{~b}$ & $6,0 \pm 0,0 \mathrm{~b}$ & $61,3 \pm 0,1 \mathrm{~b}$ & $18,0 \pm 0,5 \mathrm{a}$ & $103,5 \pm 0,0 \mathrm{a}$ \\
D & $20,0 \pm 0,7 \mathrm{a}$ & $7,6 \pm 0,0 \mathrm{a}$ & $66,8 \pm 0,0 \mathrm{a}$ & 0,00 & $94,4 \pm 0,0 \mathrm{~b}$ \\
E & $2,4 \pm 0,0 \mathrm{~d}$ & 0,00 & $10,2 \pm 0,1 \mathrm{e}$ & 0,00 & $12,6 \pm 0,0 \mathrm{e}$ \\
F & $7,7 \pm 0,0 \mathrm{c}$ & $6,3 \pm 0,0 \mathrm{~b}$ & $34,0 \pm 0,3 \mathrm{c}$ & $9,9 \pm 0,1 \mathrm{c}$ & $57,9 \pm 0,1 \mathrm{c}$ \\
\hline
\end{tabular}

*Letras diferentes na mesma coluna indicam diferença significativa a nível de $5 \%$.

A Figura 2 (B) mostra o perfil cromatográfico obtido para as isoflavonas contidas nas diferentes concentrações obtidas para a amostra manipulada pela farmácia B.

Figura 2 - Perfil cromatográfico de isoflavonas contidas em amostras manipuladas pela farmácia: (B) 1-Daidzina; 2 Glicitina; 3-Genistina; 4-Daidzeína; 5-Gliciteína e 6-Genisteína; US-Unidade de Absorbância.

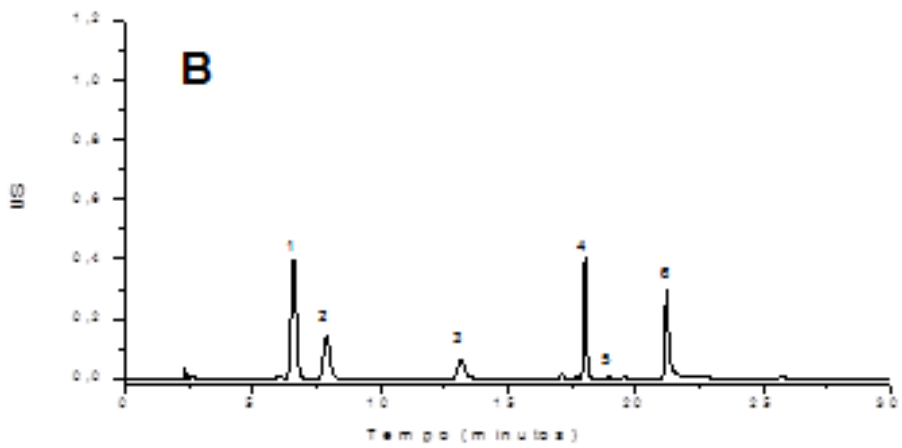

\section{Considerações Finais}

A metodologia proposta por este estudo voltado à quantificação de isoflavonas totais em extrato seco de soja e manipulados apresentou valores aceitáveis para os Fornecedores: 1, 2 e 3, ficando acima dos $40 \%$ de padronização, indicados pelos distintos fabricantes. Porém, ao se relatar teores dos compostos agliconas estudados, observa-se a distorção dos teores de isoflavonas agliconas que seriam de $40 \%$ padronizados, com valores entre 34,0\%, $12,8 \%$ e $32,4 \%$ respectivamente. No entanto, os valores encontrados nos compostos manipulados não foram 
diferentes com relação aos teores totais de isoflavonas amostras manipulaladas por farmácias A, B, C, D, E e F. A grande diferença observada neste estudo, foi a falta de padronização das matériasprimas utilizadas no comércio destes compostos. Desta forma, é importante impor medidas cautelares voltadas ao aumento da fiscalização dos fitoterápicos, especialmente quando relacionadas as isoflavonas comercializadas no Brasil para fins medicinais.

\begin{abstract}
Consumption of natural products and herbal supplements containing isoflavones have increased in the last decade, especially by the findings of the beneficial effects caused by these compounds associated with the relief of symptoms, especially those caused by menopause in women who have hormone loss. Since there are no studies reporting minimal amounts of isoflavones in glycoside forms (daidzin, genistin and glycitin) and aglycones (daidzein, glycitein and genistein), contained in dry extracts and capsules handled by pharmacies called manipulation or even official methodology for the control of quality. This study aimed to develop a quantitative method of analysis of isoflavones in soy dry extract, used as raw materials for obtaining herbal capsules sold freely without any supervision of the Brazilian trade. Quantitative analysis of isoflavones in soy dry extract was performed and manipulated using high performance liquid chromatography (HPLC) and revealed wide variations in the levels of isoflavones, depending on the vendor and manipulative. The variations occurred standardized for lots of $40 \%$ of isoflavones in $100 \mathrm{~g}$ and manipulated forms, which are marketed with labels of $20 \mathrm{mg}$ of isoflavones. The largest distortions occurred for in the values found us compounds genistein and daidzein $60.5 \%$ 451\%. Therefore, it is necessary to adopt methods of standardization and characterization of raw materials as well as certification of trading companies, since these herbal medicines are often given their consumption by medical professionals.
\end{abstract}

Key-words: Isoflavone; Dry Extracts; Manipulated.

\title{
Referências
}

BRASIL. Agência Nacional de Vigilância Sanitária. Resolução RDC n ${ }^{\circ}$ 48, de 16 de março de 2004. Dispõe sobre o registro de medicamentos fitoterápicos. Diário Oficial da União, Poder Executivo, Brasília, DF, 18 mar. 2004.

CÉSAR, I. C.; BRAGA, F. C.; SOARES, C. D. V.; NUNAN, E. A.; PIANETTI, G. A.; CONSESSA, F. A.; BARBOSA, T. A. F.; CAMPOS, L. M. M. Development and validation of a RP-HPLC method for quantification of isoflavone aglycones in hydrolyzed soy dry extracts, Journal of Chromatography B, v. 836, p. 74-78, 2006. http://dx.doi.org/10.1016/j.jchromb.2006.03.030

CÉSAR, I. C.; BRAGA, F. C.; SOARES, C. D. V.; NUNAN, E. A.; PIANETTI, G. A.; CONSESSA, F. A.; BARBOSA, T. A. F.; CAMPOS, L. M. M. Determinação de daidzeína, genisteína e gliciteína em cápsulas de isoflavonas por cromatografia em camada delgada (CCD) e cromatografia líquida de alta eficiência (CLAE). Revista Brasileira de Farmacognosia, 17, p. 616-625, 2007. http://dx.doi.org/10.1590/S0102-695X2007000400022

COWARD, L.; BARNES, N. C.; SETCHELL, K. D. R.; BARNES, S. Genistein, daidzein, and their b-glycoside conjugates: antitumor isoflavones in soybean foods from American and Asian diets, Journal of Agricultural and Food Chemistry, v. 41, p. 1961-1967, 1993. http://dx.doi.org/10.1021/jf00035a027

ELDRIDGE, C. A.; KWOLEK, F. W. Soybean Isoflavones: effects of environment and variety on composition. Journal of Agricultural and Food Chemistry, v. 31, n. 2, p. 394-396, 1983. http://dx.doi.org/10.1021/jf00116a052

GENOVESE M. I.; LAJOLO, M. F. Determinação de isoflavonas em derivados de soja. Revista Ciência e Tecnologia de Alimentos, v. 21, n. 1, p. 86-93, 2001. http://dx.doi.org/10.1590/S0101-20612001000100019

HOECK, J. A.; FEHR, W. R.; MUHRPHY, P. A.; WELKE, G. A. Influence of genotype and environment on isoflavone contents of soybean. Crop Science, v.40, p.48-51, 2000. http://dx.doi.org/10.2135/cropsci2000.40148x

LEE, S.J.; AHN, J.K.; KIM, S.H.; KIM, J.T.; HAN, S.J.; JUNG, M.Y. AND CHUNG, I.M.; Variation in Isoflavone of Soybean Cultivares with Location and Storage Duration. Journal of Agricultural and Food Chemistry, v. 51, p. 3382-3389; 2003. http://dx.doi.org/10.1021/jf0261405 
MANTOVANI, D.; CARDOZO, L.; SANTOS, C. L.; SOUZA, F. V.; WATANABE, S.C. The use of HPLC identification and quantification of isoflavones content in samples obtained in pharmacies. Acta scientiarum. Biological sciences, v. 33, n. 1, p. 7-10, 2011.

MANTOVANI, D.; CARDOZO, L.; SANTOS, C. L.; SOUZA, F. V.; WATANABE, S. C. Chromatographic Quantification of Isoflavone Content from Soy Derivates Using HPLC Technique, Journal of Chromatographic Science, v. 47, p. 766-769, 2009. http://dx.doi.org/10.1093/chromsci/47.9.766

NURMI, T.; MAZUR, W.; HEINOMEN, S.; HOKKONEN, J.; ADLERCREUTZ, H. Isoflavone content of the soy based supplements. Journal of Pharmaceutical Biomedical Analysis, v. 28, n. 1, p. 1-11, 2002. tp://dx.doi.org/10.1016/S07317085(01)00612-4

SETCHELL, K. D. R. Phytoestrogens: the biochemistry, physiology, and implications for human health of soy isoflavones. American Journal of Clinical Nutrition, v. 134, n. 6, (suppl.), p.1333S-1343S, 1998.

SETCHELL, R. D. K.; BROWN, P. M. N.; DESAI, L.; ZIMMER-NECHEMIAS, E. B.; WOLFE, T. W.; BRASHEAR, S. A.; KIRSCHNER, B.; CASSIDY, A. Bioavailability of pure isoflavones in healthy humans and analysis of commercial soy isoflavone supplements. Journal of Nutrition., v. 131, Suppl. 4, p. 1362-1375, 2001.

SONG, T. T.; HENDRICH, S.; MURPHY, A. P. Estrogenic activity of glycitein, a soy isoflavone. Journal of Agricultural and Food Chemistry, v. 47, n. 4, p. 1607-1610, 1999. http://dx.doi.org/10.1021/jf981054j

TURNER, J. V.; AGATONOVIC-KUSTRIN, S.; GLASS, B. D. Molecular aspects of phytoestrogen selective binding at estrogen receptors. Journal of Pharmaceutical Sciences's, v. 96, n. 8, p. 1879-1885, 2007. http://dx.doi.org/10.1002/jps.20987

Submetido em 07 out. 2012, Aceito para publicação em 16 jul. 2013. 